\title{
Paths and stability number in digraphs
}

\author{
Jacob Fox* Benny Sudakov ${ }^{\dagger}$ \\ Submitted: May 15, 2009; Accepted: Jul 3, 2009; Published: Jul 24, 2009 \\ Mathematics Subject Classification: 05C20, 05C38, 05C55
}

\begin{abstract}
The Gallai-Milgram theorem says that the vertex set of any digraph with stability number $k$ can be partitioned into $k$ directed paths. In 1990, Hahn and Jackson conjectured that this theorem is best possible in the following strong sense. For each positive integer $k$, there is a digraph $D$ with stability number $k$ such that deleting the vertices of any $k-1$ directed paths in $D$ leaves a digraph with stability number $k$. In this note, we prove this conjecture.
\end{abstract}

\section{Introduction}

The Gallai-Milgram theorem [7] states that the vertex set of any digraph with stability number $k$ can be partitioned into $k$ directed paths. It generalizes Dilworth's theorem [4] that the size of a maximum antichain in a partially ordered set is equal to the minimum number of chains needed to cover it. In 1990, Hahn and Jackson [8] conjectured that this theorem is best possible in the following strong sense. For each positive integer $k$, there is a digraph $D$ with stability number $k$ such that deleting the vertices of any $k-1$ directed paths in $D$ leaves a digraph with stability number $k$. Hahn and Jackson used known bounds on Ramsey numbers to verify their conjecture for $k \leq 3$. Recently, Bondy, Buchwalder, and Mercier [3] used lexicographic products of graphs to show that the conjecture holds if $k=2^{a} 3^{b}$ with $a$ and $b$ nonnegative integers. In this short note we prove the conjecture of Hahn and Jackson for all $k$.

Theorem 1 For each positive integer $k$, there is a digraph $D$ with stability number $k$ such that deleting the vertices of any $k-1$ directed paths leaves a digraph with stability number $k$.

To prove this theorem we will need some properties of random graphs. As usual, the random graph $G(n, p)$ is a graph on $n$ labeled vertices in which each pair of vertices forms an edge randomly and independently with probability $p=p(n)$.

${ }^{*}$ Department of Mathematics, Princeton, Princeton, NJ. Email: jacobfox@math.princeton.edu. Research supported by an NSF Graduate Research Fellowship and a Princeton Centennial Fellowship.

${ }^{\dagger}$ Department of Mathematics, UCLA, Los Angeles, CA 90095. Email: bsudakov@math.ucla.edu. Research supported in part by NSF CAREER award DMS-0812005 and by USA-Israeli BSF grant. 
Lemma 1 For $k \geq 3$, the random graph $G=G(n, p)$ with $p=20 n^{-2 / k}$ and $n \geq 2^{15 k^{2}}$ a multiple of $2 k$ has the following properties.

(a) The expected number of cliques of size $k+1$ in $G$ is at most $20\left(\begin{array}{c}k+1 \\ 2\end{array}\right)$.

(b) With probability more than $\frac{2}{3}$, every induced subgraph of $G$ with $\frac{n}{2 k}$ vertices has a clique of size $k$.

Proof: (a) Each subset of $k+1$ vertices has probability $p^{\left(\begin{array}{c}k+1 \\ 2\end{array}\right)}$ of being a clique. By linearity of expectation, the expected number of cliques of size $k+1$ is

$$
\left(\begin{array}{c}
n \\
k+1
\end{array}\right) p^{\left(\begin{array}{c}
k+1 \\
2
\end{array}\right)}=\left(\begin{array}{c}
n \\
k+1
\end{array}\right) 20^{\left(\begin{array}{c}
k+1 \\
2
\end{array}\right)} n^{-k-1} \leq 20^{\left(\begin{array}{c}
k+1 \\
2
\end{array}\right)} .
$$

(b) Let $U$ be a set of $\frac{n}{2 k}$ vertices of $G$. We first give an upper bound on the probability that $U$ has no clique of size $k$. For each subset $S \subset U$ with $|S|=k$, let $B_{S}$ be the event that $S$ forms a clique, and $X_{S}$ be the indicator random variable for $B_{S}$. Since $k \geq 3$, by linearity of expectation, the expected number $\mu$ of cliques in $U$ of size $k$ is

$$
\mu=\mathbb{E}\left[\sum_{S} X_{S}\right]=\left(\begin{array}{c}
\frac{n}{2 k} \\
k
\end{array}\right) p^{\left(\begin{array}{c}
k \\
2
\end{array}\right)} \geq \frac{n^{k}}{2(2 k)^{k} k !} 20^{\left(\begin{array}{c}
k \\
2
\end{array}\right)} n^{1-k} \geq 2 n .
$$

Let $\Delta=\sum \operatorname{Pr}\left[B_{S} \cap B_{T}\right]$, where the sum is over all ordered pairs $S, T$ with $|S \cap T| \geq 2$. We have

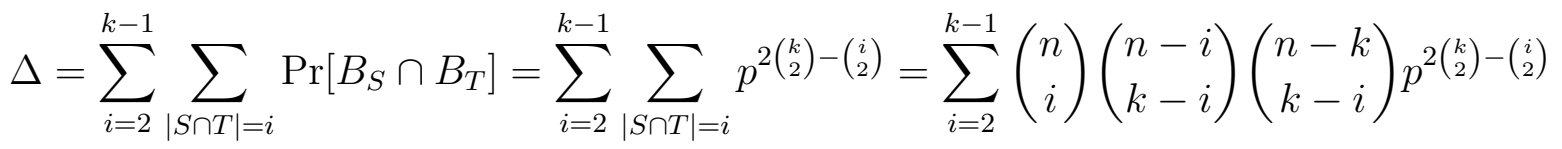

$$
\begin{aligned}
& \leq \sum_{i=2}^{k-1} n^{2 k-i} p^{k(k-1)-\left(\begin{array}{c}
i \\
2
\end{array}\right)} \leq 20^{k^{2}} \sum_{i=2}^{k-1} n^{2-i+i(i-1) / k} \leq k 20^{k^{2}} n^{2 / k}
\end{aligned}
$$

Here we used the fact that $i(i-1) / k-i$ for $2 \leq i \leq k-1$ clearly achieves its maximum when $i=2$ or $i=k-1$.

Using that $k \geq 3$ and $n \geq 2^{15 k^{2}}$, it is easy to check that $\Delta \leq n$. Hence, by Janson's inequality (see, e.g., Theorem 8.11 of [2]) we can bound the probability that $U$ does not contain a clique of size $k$ by $\operatorname{Pr}\left[\wedge_{S} \bar{B}_{S}\right] \leq e^{-\mu+\Delta / 2} \leq e^{-n}$. By the union bound, the probability that there is a set of $\frac{n}{2 k}$ vertices of $G(n, p)$ which does not contain a clique of size $k$ is at most $\left(\begin{array}{c}n \\ \frac{n}{2 k}\end{array}\right) e^{-n} \leq 2^{n} e^{-n}<1 / 3$.

The proof of Theorem 1 combines the idea of Hahn and Jackson of partitioning a graph into maximum stable sets and orienting the graph accordingly with Lemma 1 on properties of random graphs.

Proof of Theorem 1. Let $k \geq 3$ and $n \geq 2^{15 k^{2}}$. By Markov's inequality and Lemma 1 (a), the probability that $G(n, p)$ with $p=20 n^{-2 / k}$ has at most $2 \cdot 20^{\left(\begin{array}{c}k+1 \\ 2\end{array}\right)}$ cliques of size $k+1$ is at least $1 / 2$. Also, by Lemma 1 (b), we have that with probability at least $2 / 3$ every set of $\frac{n}{2 k}$ vertices of this random graph contains a clique of size $k$. Hence, with positive 
probability (at least $1 / 6$ ) the random graph $G(n, p)$ has both properties. This implies that there is a graph $G$ on $n$ vertices which contains at most $2 \cdot 20^{\left(\begin{array}{c}k+1 \\ 2\end{array}\right)}$ cliques of size $k+1$ and every set of $\frac{n}{2 k}$ vertices of $G$ contains a clique of size $k$. Delete one vertex from

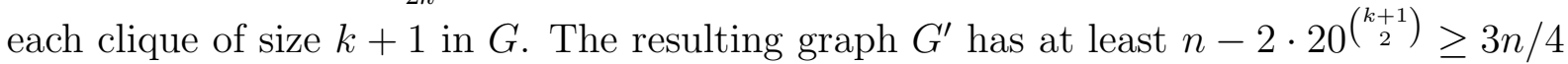
vertices and no cliques of size $k+1$. Next pull out vertex disjoint cliques of size $k$ from $G^{\prime}$ until the remaining subgraph has no clique of size $k$, and let $V_{1}, \ldots, V_{t}$ be the vertex sets of these disjoint cliques of size $k$. Since every induced subgraph of $G$ of size at least $\frac{n}{2 k}$ contains a clique of size $k$, then $\left|V_{1} \cup \ldots \cup V_{t}\right| \geq \frac{3 n}{4}-\frac{n}{2 k} \geq \frac{n}{2}$. Define the $\operatorname{digraph} D$ on the vertex set $V_{1} \cup \ldots \cup V_{t}$ as follows. The edges of $D$ are the nonedges of $G$. In particular, all sets $V_{i}$ are stable sets in $D$. Moreover, all edges of $D$ between $V_{i}$ and $V_{j}$ with $i<j$ are oriented from $V_{i}$ to $V_{j}$. By construction, the stability number of $D$ is equal to the clique number of $G^{\prime}$, namely $k$. Also any set of $\frac{n}{2 k}$ vertices of $D$ contains a stable set of size $k$. Note that every directed path in $D$ has at most one vertex in each $V_{i}$. Hence, deleting any $k-1$ directed paths in $D$ leaves at least $|D| / k \geq \frac{n}{2 k}$ remaining vertices. These remaining vertices contain a stable set of size $k$, completing the proof.

Remark. Note that in order to prove Theorem 1, we only needed to find a graph $G$ on $n$ vertices with no clique of size $k+1$ such that every set of $\frac{n}{2 k}$ vertices of $G$ contains a clique of size $k$. The existence of such graphs was first proved by Erdös and Rogers [6], who more generally asked to estimate the minimum $t$ for which there is a graph $G$ on $n$ vertices with no clique of size $s$ such that every set of $t$ vertices of $G$ contains a clique of size $r$. Since then a lot of work has been done on this question, see, e.g., [9, 1, 10, 5]. Although most results for this problem rely on probabilistic arguments, Alon and Krivelevich [1] give an explicit construction of an $n$-vertex graph $G$ with no clique of size $k+1$, such that every subset of $G$ of size $n^{1-\epsilon_{k}}$ contains a $k$-clique. Since we only need a much weaker result to prove the conjecture of Hahn and Jackson, we decided to include its very short and simple proof to keep this note self-contained.

Acknowledgments. We would like to thank Adrian Bondy for stimulating discussions and generously sharing his presentation slides. We also are grateful to Noga Alon for drawing our attention to the paper [1]. Finally, we want to thank the referee for helpful comments.

\section{References}

[1] N. Alon and M. Krivelevich, Constructive bounds for a Ramsey-type problem, Graphs Combin. 13 (1997), 217-225.

[2] N. Alon and J. H. Spencer, The Probabilistic Method, 3rd ed., Wiley, 2008.

[3] J. A. Bondy, X. Buchwalder, and F. Mercier, Lexicographic products and a conjecture of Hahn and Jackson, SIAM J. Discrete Math. 23 (2009), 882-887.

[4] R. P. Dilworth, A decomposition theorem for partially ordered sets, Ann. of Math. 51 (1950), 161-166. 
[5] A. Dudek and V. Rödl, On $K_{s}$-free subgraphs in $K_{s+k}$-free graphs and vertex Folkman numbers, submitted.

[6] P. Erdős and C. A. Rogers, The construction of certain graphs, Can. J. Math. 14 (1962), 702-707.

[7] T. Gallai and A. N. Milgram, Verallgemeinerung eines graphentheoretischen Satzes von Rédei, Acta. Sci. Math. 21 (1960) 181-186.

[8] G. Hahn and B. Jackson, A note concerning paths and independence number in digraphs, Discrete Math. 82 (1990), 327-329.

[9] M. Krivelevich, Bounding Ramsey numbers through large deviation inequalities, Random Structures Algorithms 7 (1995), 145-155.

[10] B. Sudakov, Large $K_{r}$-free subgraphs in $K_{s}$-free graphs and some other Ramsey-type problems, Random Structures Algorithms 26 (2005), 253-265. 